\section{Gesundheitsleistungen, individuelle}

T. Arndt

Bioscientia Institut für Medizinische Diagnostik GmbH, Ingelheim, Deutschland

\section{Synonym(e) IGeL}

Definition Gesundheitsleistungen, die nicht zum Leistungsumfang der gesetzlichen Krankenversicherung (GKV) gehören, dennoch von Patientinnen und Patienten nachgefragt werden, ärztlich empfehlenswert oder aufgrund des Patientenwunsches ärztlich vertretbar sind (1t. Kassenärztliche Bundesvereinigung).

Beschreibung Weiterentwicklungen in der Medizin und - Laboratoriumsmedizin finden, aus verschiedenen Gründen, keinen oder einen verzögerten Eingang in das Leistungs- spektrum der GKV. Dies kann Analyte mit nicht nachgewiesener, konträr diskutierter, aber auch mit bewiesen verbesserter diagnostischer Aussagekraft im Vergleich zu etablierten Kenngrößen betreffen.

Daneben werden einzelne Analyte, die Teil der gesetzlichen Kassenleistungen sind und Kombinationen aus Kassenleistungen und/oder IGeL in Untersuchungsprofilen wie z. B. Allergie-Panel, Arterioskleroserisikoprofil, Diabetesprofil etc. zusammengefasst, die dann vom Patienten vollständig bezahlt werden müssen. Der (unangemessene) IGeL-Einsatz wird umgangssprachlich als „IGeLn“ bezeichnet.

\section{Literatur}

Broglie MG, Pranschke-Schade S, Schade H-J (2016) Gebührenhandbuch 2016 - Kommentar für Ärzte - EBM - GOÄ - IgeL. Medical Tribune, Wiesbaden 